\title{
Características biomecânicas dos pés no período gravídico-puerperal: estudo de caso
}

\author{
Biomechanical characteristics of feet during pregnancy and puerperium: \\ a case study
}

\section{Priscilla Geraldine Wittkopf ${ }^{1} \bowtie$, Juliana Kretzer ${ }^{1}$, Deyse Machado Borges ${ }^{2}$, Gilmar Moraes Santos ${ }^{2}$, Fabiana Flores Sperandio ${ }^{2}$}

1 Mestre em Ciências do Movimento Humano pela Universidade do Estado de Santa Catarina (UDESC), Florianópolis, SC.

2 Professor(a) doutor(a) do Programa de Pós-Graduação em Fisioterapia da UDESC, Florianópolis, SC.

\section{RESUMO}

Objetivos: Avaliar as variações antropométricas e a distribuição de pressão plantar dos pés durante o ciclo gravídico-puerperal de uma paciente. Descrição do caso: Este estudo descritivo-exploratório analisou uma gestante por meio de oito avaliações, seis durante a gestação (na $26^{\mathbf{a}}$, $28^{\mathbf{a}}$, $30^{\mathrm{a}}, 32^{\mathrm{a}}, 34^{\mathrm{a}}$ e $36^{\mathrm{a}}$ semana da gestação) e duas no puerpério (10ํㅡ e $50^{\circ}$ dia após o parto). Foram avaliadas seis variáveis antropométricas dos pés e tornozelos. Por meio do escaneamento dos pés, obteve-se indiretamente o ângulo da impressão plantar. O Sistema Pedar-Mobile (Novel $\mathrm{GmbH}$ ), avaliou o índice do arco plantar e o pico de pressão máxima plantar tanto estático como dinâmico. Durante o período gestacional o comprimento e o perímetro do mediopé direito apresentaram-se maiores do que o esquerdo. Ocorreu diminuição do ângulo plantar com a evolução da gestação. A variação do índice do arco plantar na tarefa estática mostrou variação mínima em todo o período avaliado, enquanto na dinâmica demonstrou diminuição progressiva dos valores durante a gestação e aumento no período puerperal. As médias dos picos de pressão plantar na tarefa dinâmica foram mais elevadas durante o período gestacional em comparação ao puerpério.

Conclusões: Estes dados mostram alterações nas características biomecânicas dos pés no ciclo gravídico-puerperal, ressaltando aumento na perimetria e diminuição do arco plantar.

DESCRITORES: gestação; deformidades do pé; biomecânica; saúde; postura.

\section{ABSTRACT}

Aims: To evaluate anthropometric variations and distribution of plantar pressure during pregnancy and puerperium.

Case description: In this descriptive exploratory study, a pregnant woman was submitted to eight assessments, six of them during pregnancy (at 26, 28, 30,32, 34, and 36 weeks of gestation) and two in the postpartum period (at 10 and 50 days postpartum). Six foot and ankle anthropometric parameters were assessed. The footprint angle was indirectly obtained by scanning electron microscopy. The Pedar-Mobile (Novel $\mathrm{GmbH}$ ) system assessed the plantar arch index and maximum peak plantar pressure both statically and dynamically. The right mid-foot length and circumference were larger than those of the left mid-foot during the gestational period. Footprint angle decreased as the gestational period progressed. The plantar arch index in static footprints varied slightly throughout the study period, but it progressively decreased during pregnancy and increased in the postpartum period. The average peak plantar pressure in dynamic footprints was higher during pregnancy than in the postpartum period.

Conclusions: These data reveal changes in the biomechanics of feet during pregnancy and puerperium, indicating an increase in circumference measurement and a reduction in plantar arch.

KEY WORDS: pregnancy; foot deformities; biomechanics; health; posture. 


\section{INTRODUÇÃO}

O aumento da massa corporal, associado à retenção de líquido e ao aumento dos níveis do hormônio placentário relaxina em sete a dez vezes durante a gestação apresentam potencial de sobrecarga ao sistema musculoesquelético [1-3]. É possível que mecanismos patomecânicos agudos ou crônicos, vistos como desvios na artrocinemática, possam contribuir para mudanças estruturais com consequências de longo prazo $[4,5]$.

As alterações posturais são comuns no período da gestação, gerando sobrecarga e desconforto nos pés. Os ajustes determinam deformações na largura, no comprimento e, principalmente, na altura do arco plantar [6]. Além disso, podem ocorrer alterações na distribuição da pressão plantar durante tarefas, tanto estáticas quanto dinâmicas [7], o que pode comprometer a realização das atividades domésticas e laborais.

Diante de tais considerações, este estudo objetivou avaliar em conjunto as variações antropométricas e a distribuição da pressão plantar dos pés durante o ciclo gravídico-puerperal. Entende-se que esta análise poderá facilitar o reconhecimento de medidas que possam promover a saúde e o bem-estar dos pés de gestantes e puérperas.

O estudo foi aprovado pelo Comitê de Ética em Pesquisa da Universidade do Estado de Santa Catarina sob número105/2003 e cumpre os termos do Conselho Nacional de Saúde.

\section{RELATO DO CASO}

Este estudo descritivo-exploratório foi realizado com uma gestante de 31 anos de idade, com 1,63 m de estatura e $57 \mathrm{~kg}$ antes da gestação e diagnóstico nutricional de baixo peso [8]. Multípara, gesta II e intervalo interpartal de seis anos, teve controle do peso e das semanas gestacionais através da data da última menstruação e do exame ultrassonográfico mensal. A gestante aumentou o peso corporal em $12 \mathrm{~kg}$ do início ao final da gravidez. Os pesos aferidos no $10^{\circ}$ e o $50^{\circ}$ dias pós-parto foram de $4,3 \mathrm{~kg} \mathrm{e} 4 \mathrm{~kg}$, respectivamente.

Os dados a seguir foram coletados quinzenalmente, entre a $26^{\mathrm{a}}$ e a $36^{\mathrm{a}}$ semana gestacional e no $10^{\circ}$ e $50^{\circ}$ dia de puerpério. As avaliações foram realizadas no mesmo horário do dia e pelo mesmo avaliador. $\mathrm{Na}$ pré-avaliação registrou-se pressão arterial, temperatura corporal, temperatura do ambiente, tipo de calçado usado e atividades realizadas. Os procedimentos de avaliação iniciavam com a gestante deitada e com os pés elevados em aproximadamente $45^{\circ}$, por meio de uma cunha estofada, visando reduzir o edema de membros inferiores.

Em seguida realizava-se, na postura bípede, a mensuração antropométrica tendo-se como base as variáveis: comprimento do pé direito e esquerdo; perímetro da articulação metatarso-falangeana direita e esquerda; mediopé direito e esquerdo; largura da articulação metatarso-falangeana direita e esquerda; e mensuração em forma de oito do pé e tornozelo direitos e esquerdos. Foi utilizada uma balança digital com resolução de 100 gramas e amplitude de medida até 200 kg, e um estadiômetro (Balmak, Santa Bárbara d'Oeste, SP) com amplitude de medida até $2 \mathrm{~m}$ e escala de $1 \mathrm{~mm}$. Para mensuração antropométrica dos pés utilizaram-se paquímetro digital e fita métrica.

Na sequência, foi realizada a digitalização plantar, utilizando-se um Scanner TCÊ-S 430, para obtenção indireta do ângulo de pressão plantar direito e esquerdo. Os índices do arco plantar direito e esquerdo e os picos de pressão máxima plantar direita e esquerda também foram avaliados nas tarefas estáticas e dinâmicas através da distribuição de pressão plantar por meio do Sistema Pedar-Mobile (Novel GmbH, Munique, Alemanha) [9].

Utilizou-se do mesmo calçado e das mesmas palmilhas sensorizadas do Sistema Pedar-Mobile em todas as avaliações. A frequência de aquisição desse sistema foi de $50 \mathrm{~Hz}$. O pé foi dividido em quatro regiões, retropé (heel), mediopé (midfoot), antepé (forefoot) e dedos (toes). Foi permitido um período de adaptação da gestante ao sistema tanto para aquisição estática quanto para dinâmica. Para tal, foram avaliadas seis tentativas, escolhendo-se as três melhores aquisições. Os dados foram tabulados no programa Microsoft Excel e analisados por meio de estatística descritiva (média e desvio padrão).

A Tabela 1 evidencia que o comprimento dos pés manteve-se com uma pequena assimetria durante o período gestacional, ao passo que no puerpério essa tendência não se repetiu. Durante o período gestacional o comprimento do pé direito foi maior do que o esquerdo. O perímetro do mediopé direito, pé dominante, manteve-se mais elevado que o do mediopé esquerdo durante os diferentes períodos analisados.

Com relação à mensuração em forma de oito dos pés e tornozelos, observou-se que os maiores valores foram encontrados no pé direito. A variação do perímetro, assim como a largura da articulação metatarso-falangeana, apresentaram valores maiores para o pé esquerdo, diferentemente do que havia sido observado nas demais variáveis antropométricas analisadas. 
Tabela 1. Variáveis antropométricas do período gravídico-puerperal da paciente em estudo.

\begin{tabular}{|c|c|c|c|c|c|c|c|c|c|c|}
\hline \multirow{2}{*}{ Período } & \multicolumn{10}{|c|}{ Variável antropométrica (mm) } \\
\hline & CPD & CPE & PMFD & PMFE & PMD & PME & LMFD & LMFE & MFOD & MFOE \\
\hline $26^{\underline{a}}$ semana & 251,1 & 248,9 & 243,5 & 244,9 & 246,2 & 240,0 & 99,63 & 99,72 & 515,1 & 507,2 \\
\hline $28^{\underline{a}}$ semana & 250,8 & 249,8 & 245,2 & 248,1 & 245,3 & 240,1 & 98,10 & 98,20 & 510,9 & 505,7 \\
\hline $30^{\mathrm{a}}$ semana & 249,9 & 246,9 & 244,9 & 245,9 & 245,5 & 240,3 & 99,21 & 99,86 & 518,2 & 504,9 \\
\hline $32 \underline{\mathrm{a}}$ semana & 246,7 & 245,5 & 243,8 & 244,7 & 248,2 & 240,5 & 98,14 & 98,72 & 517,9 & 516,2 \\
\hline $34^{\mathrm{a}}$ semana & 246,9 & 245,7 & 242,9 & 247,2 & 248,7 & 243,1 & 97,95 & 98,70 & 516,5 & 514,4 \\
\hline $36^{\underline{a}}$ semana & 248,9 & 247,8 & 243,5 & 245,8 & 246,3 & 238,2 & 99,34 & 99,75 & 519,4 & 519,1 \\
\hline $10^{\circ}$ dia de puerpério & 244,5 & 244,6 & 239,3 & 240,3 & 244,0 & 238,6 & 97,58 & 97,64 & 510,2 & 500,9 \\
\hline $50^{\circ}$ dia de puerpério & 243,7 & 243,8 & 238,4 & 239,8 & 242,5 & 237,2 & 96,24 & 96,38 & 507,0 & 498,8 \\
\hline
\end{tabular}

CPD: comprimento do pé direito; CPE: comprimento do pé esquerdo; PMFD: perímetro da articulação metatarso-falangeana direita; PMFE: Perímetro da articulação metatarsofalangeana esquerda; PMD: perímetro do mediopé direito; PME: perímetro do mediopé esquerdo; LMFD: largura da articulação metatarso-falangeana direita; LMFE: largura da articulação metatarso-falangeana esquerda; MFOD: mensuração em forma de 8 do pé e tornozelo direito; MFOE: mensuração em forma de 8 do pé e tornozelo esquerdo.

Na Tabela 2, observa-se a diminuição do ângulo plantar com a evolução da gestação, enquanto no pósparto os valores tornaram-se relativamente mais altos. O pé esquerdo apresentou maiores valores em relação ao pé direito e essa diferença foi mantida nos diferentes períodos avaliados. A variação do índice do arco plantar na tarefa estática mostrou, tanto no pé direito quanto no esquerdo, variação mínima no período gestacional e no pós-parto. A variação do índice do arco plantar na tarefa dinâmica demonstrou diminuição progressiva dos valores durante a gestação e aumento no período puerperal.

De acordo com a Tabela 3, verificam-se os valores de média das variáveis pico de pressão plantar nas quatro áreas do pé: retropé, mediopé, antepé e dedos, para os pés esquerdo e direito, durante a tarefa dinâmica. As médias dos picos de pressão plantar na tarefa dinâmica foram mais elevadas durante o período gestacional em comparação ao puerpério, em ambos os pés.

Tabela 2. Variação do ângulo da impressão plantar, variação do índice do arco plantar estático, variação do índice do arco plantar dinâmico no período gestacional e puerpério da paciente.

\begin{tabular}{|c|c|c|c|c|c|c|}
\hline \multirow{2}{*}{ Período } & \multicolumn{2}{|c|}{ Ângulo de impressão plantar (o) } & \multicolumn{2}{|c|}{ Índice do arco plantar estático $(\stackrel{\circ}{)})$} & \multicolumn{2}{|c|}{ Índice do arco plantar dinâmico (o) } \\
\hline & Pé direito & Pé esquerdo & Pé direito & Pé esquerdo & Pé direito & Pé esquerdo \\
\hline $26^{\mathrm{a}}$ semana & 53 & 55 & 0,33 & 0,32 & 0,3 & 0,31 \\
\hline $28^{\mathrm{a}}$ semana & 53 & 55 & 0,31 & 0,32 & 0,33 & 0,32 \\
\hline $30^{\mathrm{a}}$ semana & 52 & 53 & 0,32 & 0,32 & 0,27 & 0,29 \\
\hline $32 \stackrel{\text { a }}{\text { semana }}$ & 52 & 53 & 0,32 & 0,30 & 0,25 & 0,26 \\
\hline $34^{\mathrm{a}}$ semana & 52 & 53 & 0,33 & 0,29 & 0,25 & 0,25 \\
\hline $36^{\mathrm{a}}$ semana & 52 & 53 & 0,33 & 0,34 & 0,28 & 0,29 \\
\hline $10^{\circ}$ dia de puerpério & 54 & 55 & 0,33 & 0,32 & 0,3 & 0,3 \\
\hline $50^{\circ}$ dia de puerpério & 54 & 56 & 0,35 & 0,33 & 0,26 & 0,28 \\
\hline
\end{tabular}

Tabela 3. Média dos picos de pressão plantar dos pés durante a tarefa dinâmica na gestação e no puerpério da paciente.

\begin{tabular}{l|c|c|c|c|}
\hline & \multicolumn{2}{|c|}{ Pé direito } & \multicolumn{2}{c|}{ Pé esquerdo } \\
\hline & $\begin{array}{c}\text { Período gestacional } \\
(\mathbf{k P a})\end{array}$ & $\begin{array}{c}\text { Puerpério } \\
(\mathbf{k P a})\end{array}$ & $\begin{array}{c}\text { Período gestacional } \\
(\mathbf{k P a})\end{array}$ & $\begin{array}{c}\text { Puerpério } \\
(\mathbf{k P a})\end{array}$ \\
\hline Retropé & Média & Média & Média & Média \\
\hline Mediopé & 203,33 & 189,45 & 217,4 & 190,55 \\
Antepé & 132,4 & 121,65 & 136,48 & 132,75 \\
\hline Dedos & 243,35 & 206,7 & 260,36 & 230,55 \\
\hline
\end{tabular}




\section{DISCUSSÃO}

A retenção de líquido e consequente edema, em conjunto com o crescimento do concepto e com o acúmulo de tecido adiposo, são os responsáveis pelo aumento de peso corporal e também pelo maior volume das extremidades durante a gestação [2]. A gestante deste estudo apresentou aumento de $12 \mathrm{~kg}$ do início ao final da gravidez, mantendo-se entre os limites recomendados $(11,4$ a $15,9 \mathrm{~kg})$ [10]. No puerpério da paciente, a massa corpórea relativa durante as duas avaliações foi de 4,3 kg e $4 \mathrm{~kg}$. Os valores obtidos estão dentro dos considerados por Kac et al. [11]: 4,7 kg e $4,1 \mathrm{~kg}$ no $30^{\circ}$ e $60^{\circ}$ dias pós-parto, respectivamente.

Estudos prévios já evidenciaram que o aumento de carga para os pés gera alterações anatômico-fisiológicas similares às observadas na participante deste estudo, em que as variáveis antropométricas apresentaram valores maiores durante o período gestacional $[2,5]$. Ao avaliarem 40 gestantes, Wetz et al. [12] encontraram que as medidas tanto do comprimento como da largura dos pés aumentavam significativamente durante a gestação. Por outro lado, estudos que compararam a antropometria dos pés de gestantes com grupos controles não encontraram diferença significativa entre os grupos quanto ao comprimento e à largura do antepé. Porém, as larguras do mediopé e do retropé apresentaram-se significativamente mais elevadas nas mulheres grávidas $[5,13]$.

Albino et al. [14], ao avaliaram a perimetria dos tornozelos de 13 gestantes, observaram aumento nos valores do membro não dominante, do primeiro para o terceiro trimestre gestacional. Achados semelhantes foram descritos por Wetzet al. [12], que evidenciaram aumento de $9,4 \%$ do volume dos pés das gestantes. Maiores valores na perimetria dos tornozelos e dos pés também foram observados ao final da gestação na participante do presente estudo.

A variação do índice do arco plantar pouco se alterou durante o período avaliado no caso presente, apesar de ter sido observada diminuição dos valores na tarefa dinâmica. O mesmo foi observado num estudo com 1000 sujeitos, em que os autores verificaram que o comprimento do pé, a largura do mediopé, e o índice de massa corporal correlacionaram-se negativamente com arco plantar [15].

Estudos com gestantes demonstram a mesma relação e indicam que os efeitos podem ser mantidos para além do puerpério, e que a primeira gestação parece ter mais efeito sobre esses valores [4,10]. Já Jelen et al. [16] não evidenciaram mudanças neste índice em gestantes, tanto nas tarefas estáticas quanto nas dinâmicas.

Durante a gestação, além de alterações na perimetria e antropometria dos pés, também são observadas alterações na distribuição da pressão plantar. UnluOzkan e Basgul [17] encontraram aumento da pressão plantar no antepé no último trimestre gestacional, durante tarefas estáticas e dinâmicas. Por outro lado, durante o mesmo período, Gaymer et al. [18] observaram aumento da pressão no mediopé, a qual retornou aos valores normais após a gestação.

Em postura ortostática, Ribeiro et al. [19] não observaram mudanças na pressão plantar de gestantes no último trimestre, porém durante a marcha observaram diminuição da pressão no retropé e aumento no mediopé e antepé. Os autores atribuem essa mudança à anteriorização do centro gravitacional da gestante [19]. Somado a isso, estudos apontam para correlação positiva entre aumento de peso corporal e aumento da pressão plantar $[12,20]$. Essa condição também pode ser encontrada na gestante deste estudo, já que os picos de pressão plantares estiveram elevados para as tarefas dinâmicas durante o período gestacional em comparação ao puerperal, sugerindo que as mudanças no peso possam alterar as médias de pressão.

Observou-se, no caso presente, que as medidas antropométricas evidenciaram a presença de edema gestacional. Entretanto, a relação do índice do arco plantar durante o período gravídico-puerperal foi mantida. Essa relação indica, segundo Cavanagh e Rodgers [21], que a gestante em questão apresentava arco plantar estático e dinâmico plano, e esse comportamento se manteve ao longo do terceiro trimestre de gestação ( $26^{\mathrm{a}}$ semana) e até o $50^{\circ}$ dia do puerpério. Adicionalmente, Jelen et al. [16] relataram que mulheres grávidas apresentam redução no espaço localizado abaixo do arco plantar, caracterizando o chamado "arco plano". Acredita-se que o arco plano, ou queda do arco plantar, possa influenciar no tamanho do pé. Neste sentido, Dunn et al. [10] concluíram que alterações no tamanho dos pés podem tornar-se duradouras a curto e longo prazo no puerpério, o que pode ser observado frequentemente pela mudança no tamanho do calçado para um número maior.

Este estudo de caso apresenta como limitações o fato de incluir uma única participante, avaliar um grande número de variáveis e não ter feito a comparação com os dados do primeiro trimestre gestacional. Contudo, o estudo demonstrou que as adaptações anatômico-fisiológicas da gestação podem alterar as características antropométricas e de distribuição de pressão plantar dos pés durante o período gestacional. 
Tendo em vista que as alterações nos pés das gestantes podem gerar desconfortos e disfunções posturais a longo prazo, sugere-se a realização de mais estudos que avaliem as características antropométricas dos pés de grávidas e puérperas. Tais conhecimentos poderão favorecer a compreensão das relações entre essas variáveis, podendo modificar o seu manejo na prática clínica.

\section{REFERÊNCIAS}

1. Marnach ML, Ramin KD, Ramsey PS, Song SW, Stensland JJ, An KN. Characterization of the relationship between joint laxity and maternal hormones in pregnancy. Obstet Gynecol. 2003;101(2):331-5. http://dx.doi.org/10.1016/S0029-7844(02)02447-X

2. Ireland ML, Ott SM. The effects of pregnancy on the musculoskeletal system. Clin Orthop Relat Res. 2000;(372):169-79. http://dx.doi. org/10.1097/00003086-200003000-00019

3. Borg-Stein J, Dugan SA. Musculoskeletal disorders of pregnancy, delivery and post partum. Phys Med Rehabil Clin N Am. 2007;18(3): 459-76, ix. http://dx.doi.org/10.1016/j.pmr.2007.05.005

4. Segal NA, Boyer ER, Teran-Yengle P, Glass NA, Hillstrom HJ, Yack HJ. Pregnancy leads to lasting changes in foot structure. Am J Phys Med Rehabil. 2013;92(3):232-40. http://dx.doi.org/10.1097/PHM.0b013e31827443a9

5. Butler EE, Colón I, Druzin ML, Rose J. Postural equilibrium during pregnancy: decreased stability with an increased reliance on visual cues. Am J Obstet Gynecol. 2006;195(4):1104-8. http://dx.doi.org/10.1016/j.ajog.2006.06.015

6. Borg-Stein J, Dugan SA. Musculoskeletal disorders of pregnancy, delivery and post partum. Phys Med Rehabil Clin N Am. 2007;18(3): 459-76, ix. http://dx.doi.org/10.1016/j.pmr.2007.05.005

7. To WW, Cheung W. The relationship between weight gain in pregnancy, birth-weight and postpartum weight retention. Aust N Z J Obstet Gynaecol. 1998;38(2):176-9. http://dx.doi.org/10.1111/j.1479-828X.1998.tb02996.x

8. Atalah E, Castillo C, Castro R, Aldea A. Propuesta de um nuevo estandar de evaluación nutricional en embarazadas. Rev Med Chil. 1997;125(12):1429-36.

9. Institute of Medicine. Subcommittee on nutritional status weight gain during pregnancy. Washington, DC: National Academy of Sciences; 1990.

10. Dunn J, Dunn C, Habbu R, Bohay D, Anderson J. Effect of Pregnancy and Obesity on Arch of Foot. Orthopaedic Surgery. 2012;4(2): 101-4. http://dx.doi.org/10.1111/j.1757-7861.2012.00179.x

11. Kac G, D’Aquino BMV, Valente JG. Retenção de peso ao longo de nove meses pós-parto em mulheres residentes no município do Rio de Janeiro: um estudo de seguimento. Cad Saúde Pública. 2003;19(1):149-61. http://dx.doi.org/10.1590/S0102-311X2003000700016

12. Wetz HH, Hentschel J, Drerup B, Kiesel L, Osada N, Veltmann U. Changes in shape and size of the foot during pregnancy [Article in German]. Orthopade. 2006;35(11):1124, 1126-30. http://dx.doi.org/10.1007/s00132-006-1011-1

13. Nyska M, Sofer D, Porat A, Howard CB, Levi A, Meizner I. Plantar foot pressure in pregnant women. Isr J Med Sci. 1997;33(2):139-46.

14. Albino MA, Moccellin AS, Firmento Bda S, Driusso P. Modificações da força de propulsão da marcha durante a gravidez: das alterações nas dimensões dos pés. Rev Bras Ginecol Obstet. 2011;33(7):164-9. http://dx.doi.org/10.1590/S0100-72032011000700008

15. Teyhen DS, Stoltenberg BE, Collinsworth KM, Giesel CL, Williams DG, Kardouni CH, Molloy JM, Goffar SL, Christie DS, McPoil T. Dynamic plantar pressure parameters associated with static arch height index during gait. Clin Biomech (Bristol, Avon). 2009;24(4):391-6 http://dx.doi.org/10.1016/j.clinbiomech.2009.01.006

16. Jelen K, Tetkova Z, Halounova L, Pavelka K, Koudelka T, Ruzicka P. Shape characteristics of the foot arch: Dynamics in the pregnancy period. Neuro Endocrinol Lett. 2005;26(6):752-6.

17. Karadag-Saygi E1, Unlu-Ozkan F, Basgul A. Plantar pressure and foot pain in the last trimester of pregnancy. Foot Ankle Int. 2010;31(2): 153-7. http://dx.doi.org/10.3113/FAI.2010.0153

18. Gaymer C, Whalley H, Achten J, Vatish M, Costa ML. Midfoot plantar pressure significantly increases during late gestation. Foot (Edinb). 2009;19(2):114-6. http://dx.doi.org/10.1016/j.foot.2009.02.001

19. Ribeiro AP, Trombini-Souza F, de Camargo Neves Sacco I, Ruano R, Zugaib M, João SM. Changes in the plantar pressure distribution during gait throughout gestation. J Am Podiatr Med Assoc. 2011;101(5):415-23. http://dx.doi.org/10.7547/1010415

20. Ribas SI, Guirro ECO. Análise da pressão plantar e do equilíbrio postural em diferentes fases da gestação. Rev. Bras. Fisioter. 2007;11(5) 391-6. http://dx.doi.org/10.1590/S1413-35552007000500010

21. Cavanagh PR, Rodgers MM. The arch index: a useful measure from footprints. J Biomech. 1987;20(5):547-51. http://dx.doi. org/10.1016/0021-9290(87)90255-7 